\title{
Impact of financial medication assistance on medication adherence: a systematic review
}

\author{
Anna Hung, PharmD, PhD, MS; Dan V Blalock, PhD; Julie Miller, BS; Jaime McDermott, MSN, DNP candidate; \\ Hannah Wessler, BS, MS candidate; Megan M Oakes, MS; Shelby D Reed, PhD; \\ Hayden B Bosworth, PhD; and Leah L Zullig, PhD
}

\section{What is already known about this subject}

- Prescription out-of-pocket costs can be inhibitive for patients accessing medications. Over the past two decades, financial medication assistance (FMA) has grown, and specialty pharmacy services commonly help patients apply for FMA to pay for their medications.

- A prior review evaluated the impact of manufacturer patient assistance programs (PAPs) on clinical outcomes and found positive to null effects. However, the review was restricted to PAPs and did not evaluate medication adherence or patient out-of-pocket costs.

\author{
What this study adds \\ - This is the first review synthesizing \\ the impact of FMA on medication \\ adherence. We found that FMA has \\ positive impacts on all phases of \\ medication adherence as well as \\ medication persistence over one \\ year. The implementation phase \\ of adherence was most frequently \\ reported, followed by discontinuation, \\ and then initiation. There may be \\ larger impacts on the initiation phase \\ of medication adherence compared to \\ the discontinuation or implementation \\ phases; however, this signal was \\ suggested by one study, and future \\ research is necessary to confirm this \\ finding. \\ - Impact on patient out-of-pocket \\ spending was mixed, and two of the four \\ studies reporting on costs found higher \\ out-of-pocket spending among users of \\ coupons and vouchers.
}

\section{Author affiliations}

Anna Hung, PharmD, PhD, MS; Julie Miller, BS; Hannah Wessler, BS, MS candidate; Megan M Oakes, MS; Shelby D Reed, PhD; Hayden B Bosworth, PhD; Leah L Zullig, PhD; Department of Population Health Sciences, Duke University Medical Center, Durham, North Carolina. Dan V Blalock, PhD; Hayden B Bosworth, PhD; Leah L Zullig, $\mathrm{PhD}$; Center of Innovation to Accelerate Discovery and Practice Transformation (ADAPT), Durham Veterans Affairs Health Care System, Durham, North Carolina. Dan V Blalock, PhD; Hayden B Bosworth, PhD; Department of Psychiatry and Behavioral Sciences, Duke University School of Medicine, Durham, North Carolina. Jaime McDermott, MSN, DNP candidate; Hayden B Bosworth, PhD; Duke University Hospital, Durham, North Carolina. Shelby D Reed, PhD; Hayden B Bosworth, PhD; Duke Clinical Research Institute, Duke University School of Medicine, Durham North Carolina.

AUTHOR CORRESPONDENCE: Anna Hung, 919.668.1493; anna.hung@duke.edu

J Manag Care Spec Pharm 2021;27(7):924-35

Copyright $\odot 2021$, Academy of Managed Care Pharmacy. All rights reserved.

help patients apply for such programs, has increased. The impact of FMA on medication adherence and persistence has not been synthesized.

OBJECTIVE: The primary objective of this study was to review published studies evaluating the impact of FMA on the three phases of medication adherence (initiation [or primary adherence], implementation [or secondary adherence], and discontinuation) and persistence. Among these studies, the secondary objective was to report the impact of FMA on patient out-of-pocket costs and clinical outcomes. 
METHODS: A systematic review was performed using MEDLINE and Web of Science.

RESULTS: Of 656 articles identified, eight studies met all inclusion criteria. Seven studies examined FMA for medications treating cardiovascular diseases, while one study assessed FMA for cancer medications. Among included studies, FMA had a positive impact on medication adherence or persistence, and most measured this impact over one year or less. Of the three phases of medication adherence, implementation (5 of 8 ) was most commonly reported, followed by discontinuation (3 of 8), and then initiation (1 of 8). Regarding implementation, users of FMA had a higher mean medication possession ratio (MPR) than nonusers, ranging from 7 to 18 percentage points higher. The percentage of patients who discontinued medication was 7 percentage points lower in users of FMA versus nonusers for cardiovascular disease states. In one cancer study, FMA had a larger impact on initiation than discontinuation, ie, compared to nonusers, users of FMA were less likely to abandon an initial prescription (risk ratio= $0.12,95 \%$ confidence interval [Cl]: 0.08-0.18), and this effect was larger than the decreased likelihood of discontinuing the medication (hazard ratio $=0.76,95 \% \mathrm{Cl}$ : $0.66-0.88$ ). In 3 of 8 studies reporting on medication persistence, FMA increased the odds of medication persistence for one year ranged from $11 \%$ to $47 \%$, depending on the study. In addition to adherence, half of the studies reported on FMA impacts on patient out-of-pocket costs and 3 of 8 studies reported on clinical outcomes. Impacts on patient out-of-pocket costs were mixed; two studies reported that out-of-pocket costs were higher for users of a coupon or a voucher versus nonusers, one study reported the opposite, and one study reported null effects. Impacts on clinical outcomes were either positive or null.

CONCLUSIONS: We found that FMA has positive impacts on all phases of medication adherence as well as medication persistence over one year. Future studies should assess whether FMA has differential impacts based on phase of medication adherence and report on its longer-term (ie, beyond one year) impacts on medication adherence.

Patients' out-of-pocket expenditures for medications can be prohibitive and burdensome. Nearly one in four patients with a prescription report difficulty affording the cost of their prescription medications. ${ }^{1}$ Higher patient cost-sharing is associated with decreased medication utilization and adherence across a variety of clinical areas including cancer, rheumatoid arthritis, multiple sclerosis, and type 2 diabetes. $^{2-5}$ In an effort to reduce financial strain and support adherence, health care providers (eg, prescribers, pharmacists filling the prescriptions) often look to external sources of financial medication assistance (FMA) to help patients access and take their medications as prescribed.

FMA takes many forms including manufacturer-sponsored copayment (copay) cards/coupons, patient assistance programs (PAPs), and vouchers, as well as discount cards and programs from charitable foundations. The amount of FMA provided by manufacturers in the United States has grown from \$500 million in 1998 to \$7 billion annually in $2014 .^{6-8}$ In 2014, 10 of the 15 largest charitable foundations in the United States were associated with manufacturersponsored patient assistance programs.6.9 Similarly, the use of copay cards is high, especially in drug classes with a higher proportion of branded products. For example, in 2017, 37\% of prescriptions for oral oncology drugs filled through commercial insurance used a manufacturer coupon and on average, these coupons reduced the cost of each prescription by $\$ 526 .^{10}$

A previous systematic review examined the effect of manufacturer PAPs on clinical outcomes. ${ }^{11}$ The review was restricted to a single type of FMA program (ie, PAPs), and did not report the effect on medication adherence. To fill this gap in knowledge, the primary objective of our systematic review was to report the impact of FMA on medication adherence or persistence. Among the studies included, our secondary objective was to report the impact of FMA on patient out-of-pocket costs and clinical outcomes.

\section{Methods}

We conducted a systematic literature review in accordance with PRISMA guidelines for systematic reviews and reported minimum criteria from EMERGE guidelines for medication adherence studies. ${ }^{12,13}$ We searched for studies indexed in MEDLINE (PubMed) and Web of Science (Clarivate Analytics) from inception to March 2, 2020 to identify peer-reviewed studies evaluating the impact of FMA on medication adherence or persistence. The specific search terms were developed in collaboration with a medical librarian team (Supplementary Tables 1a and $\underline{1 b}$, available in online article). Bibliographic references and the review process were managed in Covidence, which automatically removed duplicate articles. There were four multidisciplinary reviewers (LZ, DB, JM, HW) throughout the review process. First, the title and abstract of each article were reviewed for eligibility by two reviewers using a doubleblind screening process. Conflicts were discussed and voted on by a panel of three reviewers consisting of multiple disciplines- health services research, nursing, and behavioral sciences. The remaining studies were triaged for full text review. At the full text stage, two reviewers (AH, LZ, DB, JM, HW) collected information from each study.

Eligibility criteria were consistently applied across all review stages. Studies were included if they: (1) examined FMA (defined as a PAP, voucher, coupon/copay card, or discount card, or a program/ pharmacy service that helped patients apply for one of these); (2) examined the impact of 


\section{FIGURE 1 Flow Diagram}

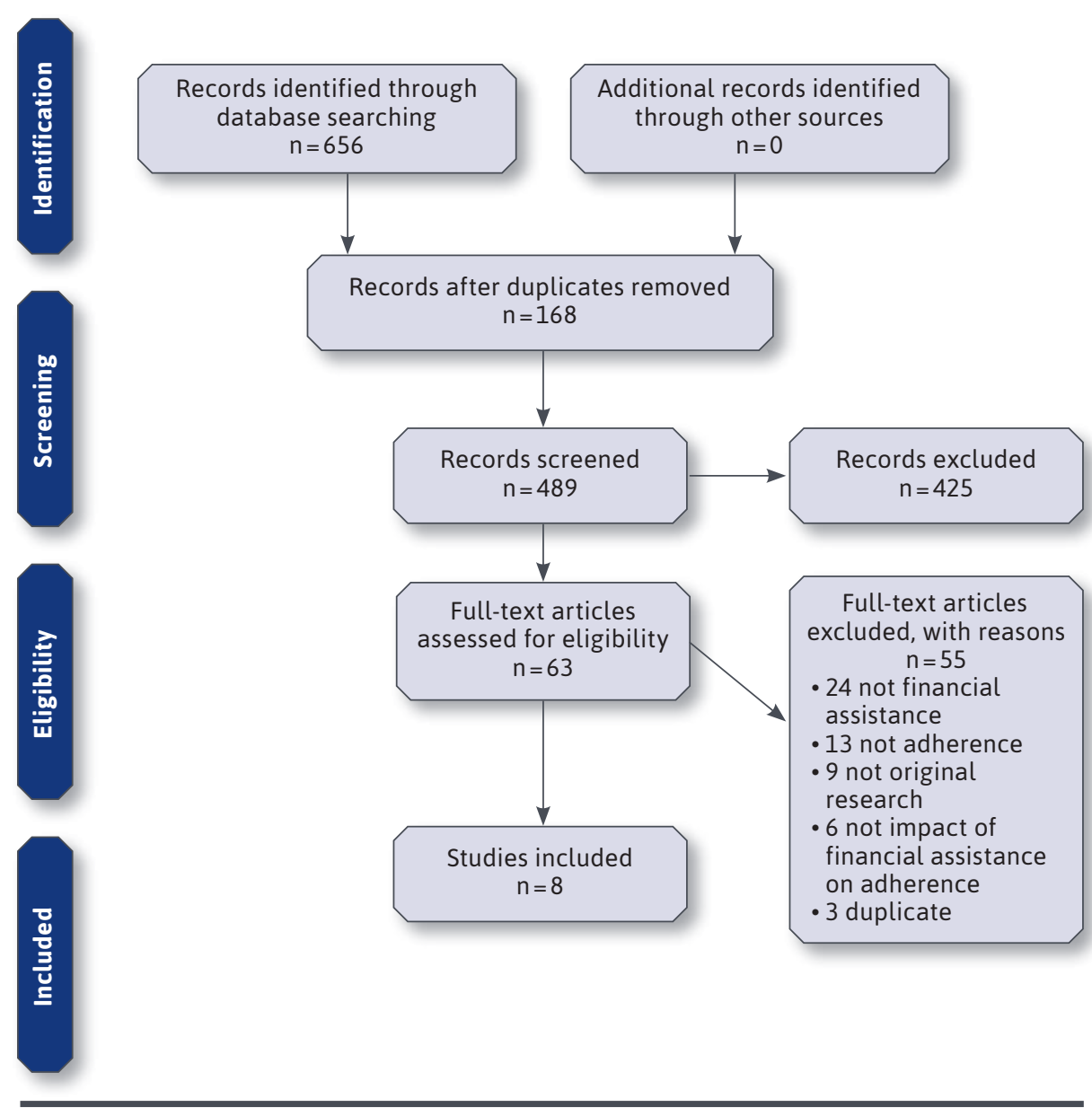

FMA on medication adherence or persistence; (3) were original research; (4) had a full text (eg, not conference abstract); and (5) were written in English. Programs or pharmacy services that only helped patients with FMA (eg, by assisting patients with applying for PAPs) were included. Programs or pharmacy services that also included clinical services, such as medication reconciliation, screening for adverse events and adherence barriers, optimizing medication therapy, and refill reminders, were excluded, due to inability to single out the impact of the financial assistance component.
Among included studies, the following data were extracted: author, publication name, journal name, study population condition, country, setting, sample size, study design, data source, demographics, description of the FMA, impact on medication adherence and persistence, impact on patient out-of-pocket costs, and impact on clinical outcomes.

When reporting impact on medication adherence, we followed the medication adherence framework by Vrijens et al and on which the EMERGE guidelines are based. ${ }^{13,14}$ In this framework, there are three different phases of medication adherence-initiation, implementation, and discontinuation. Initiation refers to when the patient takes the first dose of a prescribed medication, and in another framework by Raebel et al, is referred to as primary adherence or nonadherence, and may include measures such as abandonment of prescription (when a patient never picks up their filled medication). ${ }^{15}$ Implementation, also referred to as secondary adherence or nonadherence, ${ }^{15}$ is the extent to which a patient's actual dosing corresponds to the prescribed dosing regimen, from initiation until the last dose. This includes common measures such as medication possession ratio (MPR) and proportion of days covered (PDC). Discontinuation marks the end of therapy. In these frameworks, medication persistence is a separate construct from medication adherence, and refers to the length of time between initiation and the last dose. ${ }^{14-16}$

Two reviewers (AH, LZ) also assessed the quality of studies using the Mixed Methods Appraisal Tool (MMAT), which allows for both non-randomized and randomized studies to be evaluated in the same framework. ${ }^{17,18}$

\section{Results}

Of 656 articles identified, 425 were screened out during the abstract phase for not meeting inclusion criteria. Sixty-three full-text articles were then assessed and eight met all inclusion criteria (Figure 1). Of the eight included studies, ${ }^{19-26}$ seven took place in the United States and one took place in Australia, in a variety of settings ranging from single-site medical centers to nationwide samples (Table 1). Seven studies examined FMA for medications treating cardiovascular disease states, while one study assessed FMA for cancer medications. The majority of studies 


\section{TABLE 1 Overview of Study Population, Design, and Financial Medication Assistance}

\begin{tabular}{|c|c|c|c|c|c|}
\hline $\begin{array}{l}\text { Author year } \\
\text { (journal) }\end{array}$ & $\begin{array}{c}\text { Study population } \\
\text { condition }\end{array}$ & $\begin{array}{l}\text { Country, setting, } \\
\text { sample size }\end{array}$ & $\begin{array}{l}\text { Study design } \\
\text { (data source) }\end{array}$ & Demographics & $\begin{array}{l}\text { Description of financial } \\
\text { medication assistance }\end{array}$ \\
\hline \multicolumn{6}{|c|}{ CARDIOVASCULAR: Program/pharmacy service } \\
\hline $\begin{array}{l}\text { Marrs 2008 } \\
\text { (Pharmacotherapy) }\end{array}$ & $\begin{array}{l}\text { Dyslipidemia, } \\
\text { treated with } \\
\text { statins }\end{array}$ & $\begin{array}{l}\text { US, Single- } \\
\text { site academic } \\
\text { medical center } \\
\text { (University } \\
\text { of Colorado } \\
\text { Hospital) } \\
\mathrm{N}=240\end{array}$ & $\begin{array}{l}\text { Retrospective } \\
\text { chart review with } \\
\text { comparison group } \\
\text { (Prescription data } \\
\text { from medical center) }\end{array}$ & $\begin{array}{l}\text { Mean age } 62 \\
43 \% \text { Male }\end{array}$ & $\begin{array}{l}\text { Colorado Indigent Care } \\
\text { Program (federal- and state- } \\
\text { funded) that scales drug } \\
\text { copayment fees between } \\
\$ 0-\$ 35 \text { based on income, and } \\
\text { involves a specialist who helps } \\
\text { patients apply for a PAP }\end{array}$ \\
\hline $\begin{array}{l}\text { Schoen } 2011^{20} \\
\text { (Pharmacotherapy) }\end{array}$ & $\begin{array}{l}\text { Heart } \\
\text { disease (HTN, } \\
\text { hyperlipidemia, } \\
\text { CAD, MI, CABG, } \\
\text { HF, arrhythmia, } \\
\text { valvular heart } \\
\text { disease, other } \\
\text { comorbid } \\
\text { disease) }\end{array}$ & $\begin{array}{l}\text { US, Single-site, } \\
\text { academic inner- } \\
\text { city outpatient } \\
\text { clinic (University } \\
\text { of Illinois at } \\
\text { Chicago) } \\
\mathrm{N}=163\end{array}$ & $\begin{array}{l}\text { Prospective } \\
\text { cohort study with } \\
\text { historical control } \\
\text { as comparison } \\
\text { (Physician referral } \\
\text { documents, patient } \\
\text { self-report data) }\end{array}$ & $\begin{array}{l}\text { Mean age } 61 \\
54 \% \text { Male } \\
59 \% \text { African American } \\
24 \% \text { Hispanic } \\
13 \% \text { White } \\
4 \% \text { Other }\end{array}$ & $\begin{array}{l}\text { Pharmacy service that helps } \\
\text { patients seek financial } \\
\text { medication assistance, } \\
\text { including PAPs, state aid, and } \\
\text { samples }\end{array}$ \\
\hline \multicolumn{6}{|c|}{ CARDIOVASCULAR: Discount card } \\
\hline $\begin{array}{l}\text { Knott 201521 } \\
\text { (Health Policy) }\end{array}$ & $\begin{array}{l}\text { CVD, prescribed } \\
\text { statins }\end{array}$ & $\begin{array}{l}\text { Australia, } \\
\text { Nationwide } \\
\text { (patients of } \\
\text { providers } \\
\text { involved in } \\
\text { nationally } \\
\text { representative } \\
\text { survey) } \\
\mathrm{N}=1,260\end{array}$ & $\begin{array}{l}\text { Retrospective } \\
\text { cohort study with } \\
\text { comparison group } \\
\text { (Prescription claims } \\
\text { data from Medicare } \\
\text { Australia) }\end{array}$ & $\begin{array}{l}\text { Mean age } 68 \\
50 \% \text { Male }\end{array}$ & $\begin{array}{l}\text { Australian Pharmaceutical } \\
\text { Benefits Scheme concession } \\
\text { card for discounted out-of- } \\
\text { pocket costs for prescription } \\
\text { medications }\end{array}$ \\
\hline \multicolumn{6}{|c|}{ CARDIOVASCULAR: Manufacturer coupon } \\
\hline $\begin{array}{l}\text { Daugherty } 2013^{22} \\
(J M C P)\end{array}$ & $\begin{array}{l}\text { Incident users } \\
\text { of branded } \\
\text { statin agents } \\
\text { (atorvastatin or } \\
\text { rosuvastatin) } \\
\text { and generic } \\
\text { statin therapy } \\
\text { (lovastatin, } \\
\text { pravastatin, or } \\
\text { simvastatin) }\end{array}$ & $\begin{array}{l}\text { US, Nationwide } \\
\text { (represents } \\
40 \% \text { of retail } \\
\text { prescriptions } \\
\text { for treatment of } \\
\text { dyslipidemia) } \\
\mathrm{N}=340,350\end{array}$ & $\begin{array}{l}\text { Retrospective } \\
\text { cohort study with } \\
\text { comparison groups } \\
\text { (Retail prescription } \\
\text { claims data } \\
\text { from Symphony } \\
\text { Healthcare Solutions, } \\
\text { which identifies } \\
\text { cash claims, primary } \\
\text { and secondary } \\
\text { insurance coverage, } \\
\text { prescription } \\
\text { expenditures, and } \\
\text { coupon use) }\end{array}$ & $\begin{array}{l}\text { Mean age } 54 \\
45 \% \text { Male }\end{array}$ & $\begin{array}{l}\text { Manufacturer coupon } \\
\text { for brand-name statin } \\
\text { (atorvastatin and rosuvastatin) }\end{array}$ \\
\hline $\begin{array}{l}\text { Daubresse } 2017^{23} \\
\text { (Pharmacotherapy) }\end{array}$ & $\begin{array}{l}\text { Incident statin } \\
\text { users, within a } \\
\text { broader cohort } \\
\text { of opioid users }\end{array}$ & $\begin{array}{l}\text { US, } 11 \text { states } \\
\mathrm{N}=1,050,915\end{array}$ & $\begin{array}{l}\text { Retrospective } \\
\text { cohort study with } \\
\text { comparison groups } \\
\text { (Retail prescription } \\
\text { claims data from IMS } \\
\text { Health consisting of } \\
\text { prescriptions from } \\
\text { retail, food store, } \\
\text { independent, and } \\
\text { mass merchandiser } \\
\text { pharmacies) }\end{array}$ & $\begin{array}{l}\text { Mean age \& Male } \% \text { : } \\
\text { 1) Incident statin } \\
\text { coupon user: } 49 \text { \& } 45 \% \text {, } \\
\text { 2) Subsequent statin } \\
\text { coupon user: } 50 \text { \& } 46 \% \text {, } \\
\text { 3) Incident non-statin } \\
\text { coupon user: } 48 \text { \& } 46 \% \text {, } \\
\text { 4) Non user: } 50 \& 43 \%\end{array}$ & $\begin{array}{l}\text { Manufacturer coupon } \\
\text { for brand-name statin } \\
\text { (atorvastatin and rosuvastatin) }\end{array}$ \\
\hline
\end{tabular}




\begin{tabular}{|c|c|c|c|c|c|}
\hline $\begin{array}{c}\text { Author year } \\
\text { (journal) }\end{array}$ & $\begin{array}{c}\text { Study population } \\
\text { condition }\end{array}$ & $\begin{array}{l}\text { Country, setting, } \\
\text { sample size }\end{array}$ & $\begin{array}{l}\text { Study design } \\
\text { (data source) }\end{array}$ & Demographics & $\begin{array}{l}\text { Description of financial } \\
\text { medication assistance }\end{array}$ \\
\hline \multicolumn{6}{|c|}{ CARDIOVASCULAR: Voucher } \\
\hline $\begin{array}{l}\text { Wang } 2019^{24} \\
\text { (JAMA) }\end{array}$ & $\begin{array}{l}\text { Acute myocardial } \\
\text { infarction, } \\
\text { discharged } \\
\text { taking a P2Y12 } \\
\text { inhibitor }\end{array}$ & $\begin{array}{l}\text { US, Multi-site } \\
\text { ( } 301 \text { hospitals) } \\
N=10,102\end{array}$ & $\begin{array}{l}\text { Cluster randomized } \\
\text { clinical trial } \\
\text { (Primary measure } \\
\text { of persistence from } \\
\text { self-report data; } \\
\text { secondary measure } \\
\text { of persistence from } \\
\text { prescription claims } \\
\text { data from Symphony } \\
\text { Health) }\end{array}$ & $\begin{array}{l}\text { Median age } 62 \\
69 \% \text { Male } \\
96 \% \text { White } \\
11 \% \text { African American } \\
2 \% \text { Asian } \\
1 \% \text { American Indian or } \\
\text { Alaskan Native } \\
\text { 1\% Native Hawaiian or } \\
\text { Pacific Islander }\end{array}$ & $\begin{array}{l}\text { Vouchers that waived copays } \\
\text { of P2Y12 inhibitors for } 1 \\
\text { year to fill either a generic } \\
\text { (clopidogrel) or brand-name } \\
\text { (tigacrelor) }\end{array}$ \\
\hline $\begin{array}{l}\text { Fanaroff } 2020^{25} \\
\text { (JAMA Cardiology) }\end{array}$ & $\begin{array}{l}\text { Acute myocardial } \\
\text { infarction, } \\
\text { discharged } \\
\text { taking a P } 2 \mathrm{Y} 12 \\
\text { inhibitor }\end{array}$ & $\begin{array}{l}\text { US, Multi-site } \\
N=8,373\end{array}$ & $\begin{array}{l}\text { Post-hoc analysis of } \\
\text { cluster randomized } \\
\text { clinical trial } \\
\text { (Prescription claims } \\
\text { data from Symphony } \\
\text { Health) }\end{array}$ & $\begin{array}{l}\text { Mean age } 62 \\
68 \% \text { Male } \\
12 \% \text { Nonwhite }\end{array}$ & $\begin{array}{l}\text { Vouchers that waived copays } \\
\text { of P2Y12 inhibitors for } 1 \\
\text { year to fill either a generic } \\
\text { (clopidogrel) or brand-name } \\
\text { (tigacrelor) }\end{array}$ \\
\hline \multicolumn{6}{|c|}{ CANCER: Manufacturer copay card, discount card, or voucher } \\
\hline $\begin{array}{l}\text { Seetasith } 2019^{26} \\
\text { (Journal of Medical } \\
\text { Economics) }\end{array}$ & $\begin{array}{l}\text { ALK-positive } \\
\text { NSCLC, treated } \\
\text { with ALK } \\
\text { inhibitors }\end{array}$ & $\begin{array}{l}\text { US, Nationwide } \\
\text { (represents } \\
63 \% \text { and } 52 \% \\
\text { of prescriptions } \\
\text { dispensed } \\
\text { from retail } \\
\text { and specialty } \\
\text { pharmacies, } \\
\text { respectively) } \\
\mathrm{N}=3,143\end{array}$ & $\begin{array}{l}\text { Retrospective } \\
\text { cohort study with } \\
\text { comparison group } \\
\text { (Prescription claims } \\
\text { data from IQVIA } \\
\text { Formulary Impact } \\
\text { Analyzer database) }\end{array}$ & $\begin{array}{l}\text { Mean age } 61 \\
48 \% \text { Male }\end{array}$ & $\begin{array}{l}\text { Copay assistance: } \\
\text { manufacturer copay cards, } \\
\text { discount cards, or free-trial } \\
\text { vouchers }\end{array}$ \\
\hline
\end{tabular}

$A L K i=$ anaplastic lymphoma kinase inhibitor; $C A B G=$ coronary artery bypass graft; $C A D=$ coronary artery disease; $C l=$ confidence interval; $C V D=c a r d i o v a s c u l a r$ disease; $\mathrm{HF}=$ heart failure, $\mathrm{HTN}=$ hypertension; JAMA = Journal of the American Medical Association; JMCP= Journal of Managed Care \& Specialty Pharmacy; $\mathrm{MI}=$ myocardial infarction; $N S C L C=$ non-small cell lung cancer; $P A P=$ (manufacturer-sponsored) patient assistance program.

(5 of 8) were retrospective observational studies, while one was a prospective cohort study, one was a cluster randomized clinical trial, and one was a post-hoc analysis of the same trial. We classified the post-hoc analysis of the same trial as a separate study because the study population was a subpopulation from the trial, the study outcomes were different, and the main data source for the adherence outcome was linked pharmacy claims data (not the clinical trial data). The types of FMA included: two that were programs or pharmacy services that helped patients apply to PAPs, one that was a discount card, two that were manufacturer coupons, two that were vouchers, and one that was a mix of copay assistance, including copay cards, discount cards, and free-trial vouchers.

\section{MEDICATION ADHERENCE OR PERSISTENCE}

Among all studies, FMA had a positive impact on medication adherence or persistence (Table 2). Most measured the impact over 1 year (6 studies) or less (1 study examined impact at 6 months). The most common phase of adherence reported was implementation (5 of 8 studies; Figure 2). Among studies reporting on the implementation phase, pharmacy claims were the most commonly used data source. Specifically, MPR and PDC were used to create a dichotomous measure of adherence, with $80 \%$ as the most common threshold. Users of FMA had a higher mean MPR than nonusers, ranging from 7 to 18 percentage points higher. ${ }^{19,22}$ The percentage of patients who achieved a PDC of at least $80 \%$ ranged from 2 percentage points (not statistically significantly different, although this was for secondary medications that were not accounted for by the voucher) to 5 percentage points higher in users of FMA versus nonusers. ${ }^{21,25}$

Discontinuation was the second most common phase of adherence that was reported (3 of 8 studies). The percentage of patients who discontinued medication was 7 percentage points lower in users of FMA versus nonusers for cardiovascular disease states. ${ }^{21,23}$ Persistence was also reported in 3 of 8 studies. FMA increased odds of 


\section{TABLE 2 Impact of Financial Medication Assistance on Adherence or Persistence}

\begin{tabular}{|c|c|c|c|}
\hline $\begin{array}{c}\text { Author year } \\
\text { (journal) }\end{array}$ & $\begin{array}{c}\text { Phase of adherence } \\
\text { or persistence }\end{array}$ & Measure & Impact on adherence or persistence \\
\hline \multicolumn{4}{|c|}{ CARDIOVASCULAR: Program/pharmacy service } \\
\hline $\begin{array}{l}\text { Marrs } 2008^{19} \\
\text { (Pharmacotherapy) }\end{array}$ & Implementation & $\begin{array}{l}\text { Mean } M P R, \% \text { patients } \\
\text { with } M P R \geq 75 \% \\
\text { (over } 12 \text { months) }\end{array}$ & $\begin{array}{l}\text { Mean MPR for indigent intervention (participated in Colorado Indigent } \\
\text { Care Program) group }(0.64, S D=0.32) \text { was higher than for insured } \\
\text { (comparator) group }(0.46, S D=0.24 ; P=0.0013) \text {. } \\
\% \text { patients with } M P R \geq 75 \% \text { for indigent intervention group }(48.5 \%) \text { was } \\
\text { higher than for insured group }(15 \% ; P<0.0001) \text {. }\end{array}$ \\
\hline $\begin{array}{l}\text { Schoen } 2011^{20} \\
\text { (Pharmacotherapy) }\end{array}$ & Implementation & $\begin{array}{l}\text { Self-report } \\
\text { (at } 6 \text { months) }\end{array}$ & $\begin{array}{l}\text { Patient drug adherence improved from } 48.5 \% \text { at baseline to } 72.7 \% \text { at } \\
6 \text { months }(P<0.001) \text { after intervention (participation in pharmacy } \\
\text { service that helps patients find financial medication assistance). }\end{array}$ \\
\hline \multicolumn{4}{|c|}{ CARDIOVASCULAR: Discount card } \\
\hline $\begin{array}{l}\text { Knott } 2015^{21} \\
\text { (Health Policy) }\end{array}$ & $\begin{array}{l}\text { Implementation } \\
\text { and discontinuation }\end{array}$ & $\begin{array}{l}\% \text { who discontinued } \\
\text { (no gap defined), \% } \\
\text { who failed adherence } \\
\text { (PDC }<80 \% \text { ) } \\
\text { (over } 12 \text { months) }\end{array}$ & $\begin{array}{l}10 \% \text { of concession (discount card) users versus } 17 \% \text { of general users } \\
\text { discontinued statin therapy. } \\
8 \% \text { of concession users versus } 13 \% \text { of general users failed to adhere to } \\
\text { statin therapy. } \\
\text { In adjusted analyses, general users had a higher hazard (aHR }=1.63 \text {, } \\
95 \% \mathrm{Cl}: 1.14-2.33 \text { ) of discontinuing use and were more likely } \\
\text { (aOR }=1.60,95 \% \mathrm{Cl}: 1.04-2.44) \text { likely to fail to adhere to statin therapy } \\
\text { compared to concession users. }\end{array}$ \\
\hline \multicolumn{4}{|c|}{ CARDIOVASCULAR: Manufacturer coupon } \\
\hline $\begin{array}{l}\text { Daugherty } 2013^{22} \\
(J M C P)\end{array}$ & Implementation & $\begin{array}{l}\text { Mean MPR, \% of patients } \\
\text { with } M P R>80 \% \\
\text { (over } 1 \text { year) }\end{array}$ & $\begin{array}{l}\text { Mean MPR was highest for coupon users, slightly lower for patients } \\
\text { initiating generic statins, and lowest for noncoupon users }(61.1 \% \text { vs. } \\
60.1 \% \text { vs. } 53.8 \% ; P<0.001) \text {. } \\
\text { The } \% \text { of patients with MPR }>80 \% \text { was highest for generic users } \\
(38.4 \%) \text {, next highest for brand coupon users }(36.7 \%) \text {, and lowest for } \\
\text { brand non-coupon users }(30.4 \%) \text {. }\end{array}$ \\
\hline $\begin{array}{l}\text { Daubresse } 2017^{23} \\
\text { (Pharmacotherapy) }\end{array}$ & Discontinuation & $\begin{array}{l}\text { Cumulative probability } \\
\text { of terminating statin } \\
\text { use (defined as 6-month } \\
\text { period without statin } \\
\text { use) }\end{array}$ & $\begin{array}{l}\text { Compared to noncoupon users, initial statin coupon users were } 6.9 \% \\
\text { less likely to terminate statin therapy }(31.3 \% \text { vs } 39.2 \%, P<0.0001) \text { at } \\
\text { year } 1 \text {, and } 10.5 \% \text { less likely to terminate statin therapy }(50.6 \% \text { vs } \\
60.7 \% ; P<0.0001) \text { at year } 4 \text {. Subsequent statin coupon users were even } \\
\text { less likely to terminate statin therapy at year } 1(8.3 \%) \text { and } 4(25.9 \%) \text {. } \\
\text { Higher levels of coupon use resulted in a lower probability of } \\
\text { termination. At } 3 \text { years, those who used a coupon on only the first fill } \\
\text { were } 0.6 \% \text { more likely to terminate statin therapy than noncoupon } \\
\text { users }(57.0 \% \text { vs } 56.4 \%) \text {, whereas those who used a coupon for } 5+\text { fills } \\
\text { were } 27.9 \% \text { less likely to terminate statin therapy than noncoupon } \\
\text { users }(27.2 \% \text { vs } 56.4 \%) \text {. }\end{array}$ \\
\hline \multicolumn{4}{|c|}{ CARDIOVASCULAR: Voucher } \\
\hline $\begin{array}{l}\text { Wang } 2019^{24} \\
\text { (JAMA) }\end{array}$ & Persistence & $\begin{array}{l}\% \text { persistent at one year } \\
\text { (cannot have gap } \geq 30 \\
\text { days) through both self- } \\
\text { report and prescription } \\
\text { claims data (over } 1 \text { year) }\end{array}$ & $\begin{array}{l}\text { Patients in the intervention group (randomized to a hospital that } \\
\text { received P2Y12 inhibitor vouchers to provide to their patients) were } \\
\text { more likely to report persistence with P2Y12 inhibitor therapy than } \\
\text { patients in the usual care group ( } 87.0 \% \text { vs } 83.8 \%, P<0.001 \text {; observed } \\
\text { increase, } 3.3 \% \text { [ } 95 \% \mathrm{Cl}: 1.0-5.5]) \text {. The intervention significantly } \\
\text { increased persistence after adjusting for differences in patient } \\
\text { characteristics between groups, with an adjusted odds ratio (OR) of } \\
1.19 \text { ( } 95 \% \mathrm{Cl}: 1.02-1.40) \text {. } \\
\text { Prescription claims-defined persistence rates remained higher among } \\
\text { patients in the intervention group than in the usual care group ( } 55.2 \% \\
\text { vs } 46.3 \%, P<0.001 \text {; adjusted OR, } 1.47 \text { [ } 95 \% \mathrm{Cl}: 1.29-1.66]) \text {. } \\
\text { Within the intervention group, rates of medication persistence differed } \\
\text { substantially between patients who did and did not use the } \\
\text { co-payment voucher, whether measured by patient report }(90.0 \% \text { vs } \\
79.4 \%, P<0.001 \text { ) or pharmacy fill supply }(59.8 \% \text { vs } 43.0 \%, P<0.001) \text {. }\end{array}$ \\
\hline
\end{tabular}




\begin{tabular}{|c|c|c|c|}
\hline $\begin{array}{l}\text { Author year } \\
\text { (journal) }\end{array}$ & $\begin{array}{c}\text { Phase of adherence } \\
\text { or persistence }\end{array}$ & Measure & Impact on adherence or persistence \\
\hline $\begin{array}{l}\text { Fanaroff } 2020^{25} \\
\text { (JAMA Cardiology) }\end{array}$ & $\begin{array}{l}\text { Persistence and } \\
\text { implementation }\end{array}$ & $\begin{array}{l}\% \text { persistent at one year } \\
\text { (cannot have gap } \geq 30 \\
\text { days), } \% \text { with PDC } \geq 80 \% \\
\text { (over } 1 \text { year) }\end{array}$ & 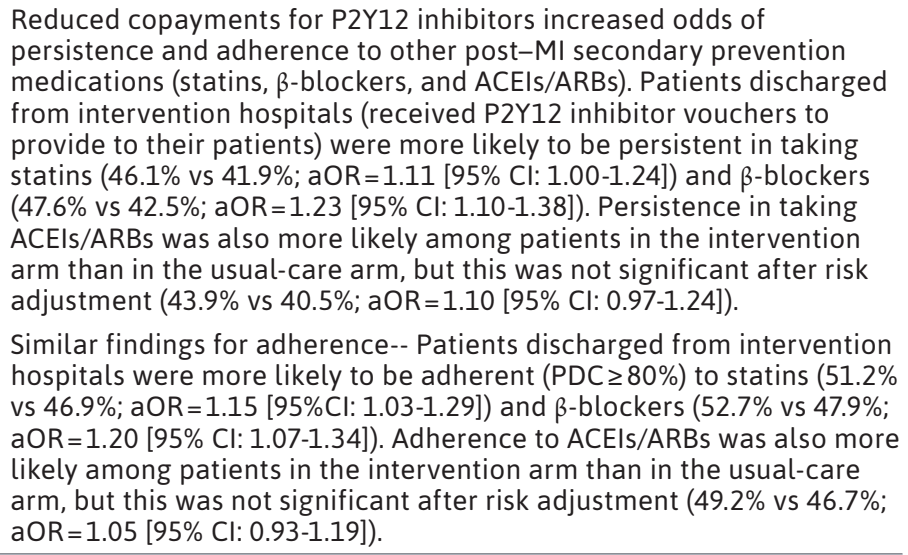 \\
\hline \multicolumn{4}{|c|}{ CANCER: Manufacturer copay card, discount card, or voucher } \\
\hline $\begin{array}{l}\text { Seetasith } 2019^{26} \\
\text { (Journal of Medical } \\
\text { Economics) }\end{array}$ & $\begin{array}{l}\text { Initiation, } \\
\text { persistence, and } \\
\text { discontinuation }\end{array}$ & $\begin{array}{l}\% \text { who abandoned } \\
\text { prescription, time } \\
\text { to picking up initial } \\
\text { prescription, persistence } \\
\text { (gap cannot be } \\
>60 \text { days), \% who } \\
\text { discontinued } \\
\text { (gap }>60 \text { days) } \\
\text { (over } 1 \text { year) }\end{array}$ & $\begin{array}{l}\text { Patients with copay assistance were less likely to abandon their } \\
\text { initial ALKi prescription than patients without copay assistance }(4.3 \% \\
\text { vs } 33.3 \%, P<0.001) \text {. In the adjusted analysis, patients with copay } \\
\text { assistance had an } 88.2 \% \text { lower risk of abandoning their first approved } \\
\text { claim (RR }[95 \% \mathrm{Cl}]=0.12[0.08-0.18]) \text {. Furthermore, for patients who } \\
\text { picked up their } \mathrm{ALKi} \text { prescription (paid claim), the time from the index } \\
\text { claim to the first picked up prescription was shorter for patients with } \\
\text { copay assistance compared to patients without copay assistance } \\
\text { (unadjusted mean [SD] days }=2.6[28.7] \text { vs } 25.7[115.3], P<0.001) \text {. } \\
\text { Patients with copay assistance were more likely to remain on their first } \\
\text { ALKi over the } 1 \text {-year follow-up compared to patients without copay } \\
\text { assistance (median days on treatment }[95 \% \mathrm{Cl}]=183[158-215] \text { vs } 140 \\
[115-154] \text {; log-rank test } P<0.001) \text {. } \\
\text { In the adjusted analysis, patients with copay assistance had a } 24.3 \% \\
\text { lower risk of } \mathrm{ALKi} \text { discontinuation than patients without copay } \\
\text { assistance }(\mathrm{HR}[95 \% \mathrm{CI}]=0.76[0.66-0.88]) \text {. }\end{array}$ \\
\hline
\end{tabular}

$A L K i=$ anaplastic lymphoma kinase inhibitor; $\mathrm{Cl}=$ confidence interval; $\mathrm{HR}=$ hazard ratio; JAMA = Journal of the American Medical Association; JMCP= Journal of Managed Care \& Specialty Pharmacy; $M P R=$ medication possession ratio; $P D C=$ proportion of days covered; $R R=$ risk ratio; $S D=s t a n d a r d$ deviation.

medication persistence for one year (with no more than a gap in medication of 29 days) by a range of $11 \%$ to $47 \% .^{24,25}$

One cancer study reported on the initiation and discontinuation phases of adherence, as well as persistence. FMA had a larger impact on initiation (eg, not picking up, or abandoning, an initial fill of a prescription) than discontinuation. Specifically, compared to nonusers, users of FMA were less likely to abandon an initial prescription, and this effect was larger than the decreased likelihood of discontinuing the medication (risk ratio of prescription abandonment $=0.12,95 \%$ confidence interval $[\mathrm{CI}]$ : 0.08-0.18 versus hazard ratio of prescription discontinuation $=0.76$, 95\% CI: 0.66-0.88). ${ }^{26}$

\section{PATIENT OUT-OF-POCKET COSTS AND CLINICAL OUTCOMES}

In addition to adherence, four of the studies reported on FMA impacts on patient out-of-pocket costs (Table 3). Impacts on patient out-of-pocket costs were mixed-two studies reported that out-of-pocket costs were higher for users of a coupon (in those with private insurance) ${ }^{22}$ or a voucher (in those with private or public insurance ${ }^{25}$ versus nonusers, while one study (in those with private, public, or other insurance) reported the opposite ${ }^{26}$ and the fourth study reported null effects (in those with either private insurance or no insurance). ${ }^{23}$ Among the two studies that reported that out-of-pocket costs were higher among users of FMA versus nonusers, impacts ranged from $\$ 25$ per year 


\section{FIGURE 2 Number of Studies That Reported on Each Phase of Medication Adherence or Persistence ${ }^{a}$}

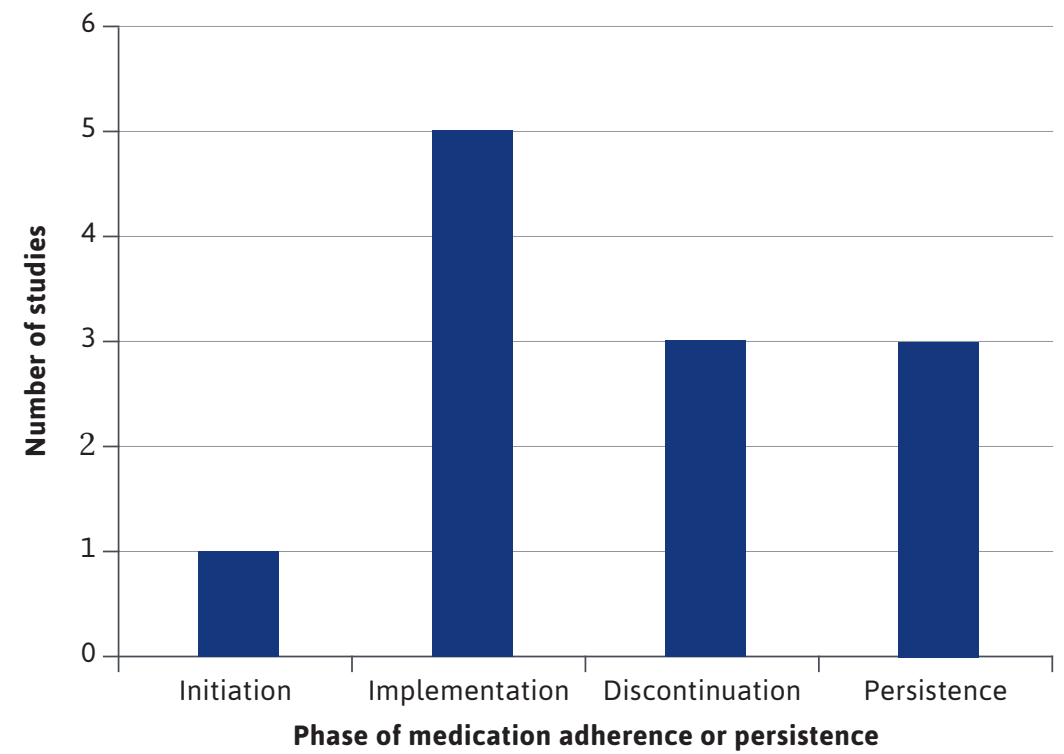

aThe total is greater than eight because each study could report more than one phase of medication adherence or persistence.

higher when comparing brand-name coupon versus non-coupon users to \$141 per year higher when comparing brand-name coupon users to generic users. ${ }^{22}$ Meanwhile, the one study that reported lower out-of-pocket costs for users of FMA versus nonusers reported a large difference of $\$ 1,179^{26}$; however, this study only reported cost differences for the first prescription claim, while all other studies reported total costs or average monthly costs over 12 months.

Three of the eight studies reported on the impact of FMA on clinical outcomes, such as low-density lipoprotein (LDL) cholesterol, international normalized ratio (INR) in warfarin users, blood pressure, hospitalizations, and major adverse cardiovascular events (MACE). Results were mixed, varying from positive effects on LDL, INR, diastolic blood pressure, and hospitalizations, ${ }^{20}$ to statistically non-significant effects on LDL, systolic blood pressure, and MACE. ${ }^{19,20,24}$

The eight studies met most (at least four of the five) MMAT methodological quality criteria (Supplementary Table 2, available in online article). Seven of eight studies had comparison groups, while the eighth study used its historical control as the comparison. The biggest concern was that four of the eight studies did not fully account for confounding in either study design or analysis.

\section{Discussion}

We found that FMA, including PAPs, vouchers, coupons/copay cards, and discount cards, as well as programs/ pharmacy services that help patients apply, had a positive impact on medication adherence across all phases, as well as medication persistence, over one year. FMA also had either positive or no effects on clinical outcomes, which was similar to a prior review, which examined the impact of PAPs on clinical outcomes, but not medication adherence or patient out-of-pocket spending. ${ }^{11}$ To our knowledge, this is the first review examining the impact of different types of FMA on medication adherence.

When reporting on medication adherence, the majority focused on the implementation phase, used either PDC or MPR as the measure, and commonly applied an $80 \%$ threshold. This is not surprising given that this threshold has been commonly used in the past to define adequate adherence in trials and quality measures for medication adherence. ${ }^{27,28}$ However, different thresholds are likely needed depending on the medication and disease state, and a binary measure may not always be appropriate..$^{28,29}$ The discontinuation phase of medication adherence, as well as medication persistence, were also reported; however, these were only measured over one year. Future studies should examine longer-term effects of FMA. Only one study reported on prescription abandonment (the initiation phase of medication adherence), and the effect size was numerically larger than that of medication discontinuation. Given this was based on only one study, whether FMA truly has a larger impact on prescription abandonment versus discontinuation or implementation is unknown and should be explored in the future.

Two studies found that users of FMA were more likely to have higher out-of-pocket costs than nonusers. This was unexpected given that cost is the medication adherence barrier that is being addressed. However, it is likely that the increased out-of-pocket costs were due to the positive impact that FMA had on medication adherence and therefore medication utilization. 


\section{TABLE 3 Impact of Financial Medication Assistance on Patient Out-of-Pocket Costs and Clinical Outcomes}

\begin{tabular}{|c|c|c|}
\hline $\begin{array}{l}\text { Author year } \\
\text { (journal) }\end{array}$ & Impact on patient out-of-pocket costs & Impact on clinical outcome \\
\hline \multicolumn{3}{|c|}{ CARDIOVASCULAR: Program/pharmacy service } \\
\hline $\begin{array}{l}\text { Marrs } 2008^{19} \\
\text { (Pharmacotherapy) }\end{array}$ & Not reported & $\begin{array}{l}\text { LDL: Proportion of patients reaching LDL goals in the } \\
\text { indigent intervention group }(68.9 \%) \text { were not significantly } \\
\text { greater than the insured group }(78.4 \% ; P=0.34) \text {. }\end{array}$ \\
\hline \multirow[t]{5}{*}{$\begin{array}{l}\text { Schoen } 2011^{20} \\
\text { (Pharmacotherapy) }\end{array}$} & \multirow[t]{5}{*}{ Not reported } & $\begin{array}{l}\text { INR: In patients receiving warfarin, mean INR increased } \\
\text { from } 2.44 \pm 0.64 \text { at baseline to } 2.61 \pm 0.53 \text { at } 6 \text { months } \\
(P<0.05) \text {. }\end{array}$ \\
\hline & & $\begin{array}{l}\text { SBP/DBP: In patients with hypertension, mean blood } \\
\text { pressure decreased from } 138 \pm 20 / 80 \pm 11 \mathrm{~mm} \mathrm{Hg} \text { at baseline } \\
\text { to } 138 \pm 19 / 78 \pm 12 \mathrm{~mm} \mathrm{Hg} \text { at } 6 \text { months }(P<0.05 \text { for diastolic } \\
\text { blood pressure only). }\end{array}$ \\
\hline & & $\begin{array}{l}\text { LDL: The mean LDL level for patients on lipid-lowering } \\
\text { drugs significantly decreased from } 126 \pm 39 \mathrm{mg} / \mathrm{dl} \text { at } \\
\text { baseline to } 108 \pm 38 \mathrm{mg} / \mathrm{dl} \text { at } 6 \text { months }(P<0.001) \text {. }\end{array}$ \\
\hline & & $\begin{array}{l}\text { For each disease measure, the improved disease control } \\
\text { seen at } 6 \text { months persisted throughout } 24 \text { months of } \\
\text { follow-up. }\end{array}$ \\
\hline & & $\begin{array}{l}\text { Hospitalization: Hospitalizations for the entire cohort } \\
\text { decreased from } 85 \text { at baseline to } 49 \text { at } 6 \text { months. }\end{array}$ \\
\hline \multicolumn{3}{|c|}{ CARDIOVASCULAR: Discount card } \\
\hline $\begin{array}{l}\text { Knott } 2015^{21} \\
\text { (Health Policy) }\end{array}$ & Not reported & Not reported \\
\hline \multicolumn{3}{|c|}{ CARDIOVASCULAR: Manufacturer coupon } \\
\hline $\begin{array}{l}\text { Daugherty } 2013^{22} \\
(J M C P)\end{array}$ & $\begin{array}{l}\text { Mean costs over } 12 \text { months } \\
\text { Brand coupon users: } \$ 194 \text { (post-coupon) } \\
\text { Brand noncoupon users: } \$ 169(P<0.001) \\
\text { Generic users: } \$ 53 \text { ( } P \text { value not reported) }\end{array}$ & Not reported \\
\hline $\begin{array}{l}\text { Daubresse } 2017^{23} \\
\text { (Pharmacotherapy) }\end{array}$ & $\begin{array}{l}\text { All coupon users had consistently lower OOP costs than } \\
\text { noncoupon users. At } 1 \text { year, average monthly out-of-pocket } \\
\text { costs for statins appeared } \$ 5 \text { lower for initial coupon users } \\
\text { than for noncoupon users; however, this difference was } \\
\text { not statistically significant (\$9.7 vs } \$ 15.9 \text {, not significant). } \\
\text { Between } 2 \text { and } 4 \text { years of follow-up, this difference } \\
\text { persisted, ranging from } \$ 2 \text { to } \$ 6 \text {. }\end{array}$ & Not reported \\
\hline
\end{tabular}

Additionally, this finding was based on a study examining a manufacturer coupon, as well as a study examining adherence and costs for adjunct medications that were not covered by the voucher but were recommended to be given with the medication covered by the voucher. Furthermore, these studies were in broad populations (and not limited to low-income populations). Nonetheless, this broad population is a limitation of using only administrative claims data to understand medication adherence barriers-other non-financial barriers, such as transportation issues may be missed. Future research on whether this finding that FMA was related to increase cost holds true using a variety of data sources, and how results vary by type of FMA and population, is warranted.

Current debate exists around some types of FMA, such as manufacturer-sponsored assistance (eg, PAPs, coupons/ copay cards). Proponents argue that manufacturer-sponsored assistance helps decrease cost-related medication nonadherence, while critics argue that manufacturersponsored assistance encourages patients to use more expensive, brand-name medications, is often time-limited (many cannot be used for more than a year), increases both patient out-of-pocket spending as well as payer spending, and leads to higher premiums for all beneficiaries. ${ }^{6,22,23,30,31} \mathrm{As}$ 


\section{TABLE 3}

Impact of Financial Medication Assistance on Patient Out-of-Pocket Costs and Clinical Outcomes (continued)

\begin{tabular}{|c|c|c|}
\hline $\begin{array}{l}\text { Author year } \\
\text { (journal) }\end{array}$ & Impact on patient out-of-pocket costs & Impact on clinical outcome \\
\hline \multicolumn{3}{|c|}{ CARDIOVASCULAR: Voucher } \\
\hline $\begin{array}{l}\text { Wang } 2019^{24} \\
\text { (JAMA) }\end{array}$ & Not reported & $\begin{array}{l}\text { MACE: There was no significant difference in MACE at } \\
1 \text { year between intervention (randomized to a hospital } \\
\text { that received P2Y12 inhibitor vouchers to provide to their } \\
\text { patients) and usual care groups (unadjusted cumulative } \\
\text { incidence, } 10.2 \% \text { vs } 10.6 \% ; P=0.65 \text {; adjusted difference, } \\
0.66 \%[95 \% \mathrm{Cl},-0.73 \% \text { to } 2.06 \%] \text {; adjusted hazard ratio, } 1.07 \\
{[95 \% \mathrm{Cl}, 0.93 \text { to } 1.25] \text { ). }}\end{array}$ \\
\hline $\begin{array}{l}\text { Fanaroff } 2020^{25} \\
\text { (JAMA Cardiology) }\end{array}$ & $\begin{array}{l}\text { Over the 1-year study period, patients in the intervention } \\
\text { arm (randomized to a hospital that received P2Y12 } \\
\text { inhibitor vouchers to provide to their patients) had higher } \\
\text { median (IQR) monthly OOP costs for all filled prescriptions } \\
\text { than patients receiving usual care (median [IQR], } \$ 26.99 \\
\text { [\$9.46-\$62.12] vs } \$ 24.36[\$ 7.78-\$ 55.21] ; P<0.001) \text {. }\end{array}$ & Not reported \\
\hline \multicolumn{3}{|c|}{ CANCER: Manufacturer copay card, discount card, or voucher } \\
\hline $\begin{array}{l}\text { Seetasith } 2019^{26} \\
\text { (Journal of Medical } \\
\text { Economics) }\end{array}$ & $\begin{array}{l}\text { Patients with copay assistance had lower mean OOP costs } \\
\text { for their first approved ALKi than patients without copay } \\
\text { assistance (mean }[S D]=\$ 26[\$ 229] \text { vs } \$ 1,205[\$ 3,543] \\
P<0.001) \text {. }\end{array}$ & Not reported \\
\hline
\end{tabular}

a result, payers have begun to modify their pharmacy benefits to include copay accumulator programs, which prevent copay assistance payments from counting towards a patient's deductible. ${ }^{32,33}$ Such programs could decrease the use of FMA if individuals expect their FMA to help them meet their deductible. Future studies should examine medication adherence and persistence over longer time periods (beyond one year after a patient is no longer eligible for the same coupon) and assess how these copay accumulator programs affect the impact of manufacturer-sponsored assistance on medication adherence.

\section{LIMITATIONS}

This review is limited by the small number of studies available that met all inclusion and exclusion criteria, as well as the quality of the studies included in this review. The large majority of these studies examined FMA for medications with cardiovascular indications and were non-randomized studies. Although we descriptively summarized the impact of FMA on medication adherence, there was a wide variety of interventions included, and the measures, as well as operationalization of measures, differed across studies.

\section{Conclusions}

This review found that FMA has positive impact on all phases of medication adherence as well as medication persistence over one year. One study found larger impacts on the initiation phase of medication adherence compared to the discontinuation phase. Impact on patient out-of-pocket spending was mixed, and two of the four studies reporting on costs found higher out-of-pocket spending among users of coupons and vouchers. In the few studies reporting impact of FMA on clinical outcomes, effects were positive or null. Future studies should assess whether FMA has differential impacts on phase of medication adherence and report on its longer-term (ie, beyond one year) impacts on medication adherence and persistence.

\section{DISCLOSURES}

This work was sponsored by a grant from Pharmaceutical Research and Manufacturers of America (PhRMA). PhRMA had no role in study design, data collection and analysis, decision to publish, or preparation of the manuscript. Hung reports past employment by Blue Cross Blue Shield Association and CVS Health and a grant from PhRMA outside of the submitted work. Zullig reports research funding from Proteus Digital Health and the PhRMA Foundation. consulting fees from Novartis. Reed reports receiving research support from Abbott Vascular, AstraZeneca, Janssen Research \& Development, Monteris, PhRMA Foundation, and TESARO and 
consulting fees from Sanofi/Regeneron, NovoNordisk, SVC Systems, and Minomic International, Inc. Bosworth reports research grants from the PhRMA Foundation, Proteus Digital Health, Otsuka, Novo Nordisk, Sanofi, Improved Patient Outcomes, Boehinger Ingelheim, $\mathrm{NIH}$, and VA, as well as consulting fees from Sanofi, Novartis, Otsuka, Abbott, Xcenda, Preventric Diagnostics, and the Medicines Company. The other authors have nothing to report.

This work was presented as a poster presentation at the ESPACOMP Annual Meeting in November 2020.

\section{ACKNOWLEDGMENTS}

The authors thank the Duke medical librarians for their support. The authors also thank the Center of Innovation to Accelerate Discovery and Practice Transformation (ADAPT) at the Durham Veterans Affairs Health Care System for its support. The content of this study is solely the responsibility of the authors and does not necessarily reflect the position or policy of Duke University, the U.S. Department of Veterans Affairs, or the U.S. government.

\section{REFERENCES}

1. Poll: Nearly 1 in 4 Americans Taking Prescription Drugs Say It's Difficult to Afford Their Medicines, including Larger Shares Among Those with Health Issues, with Low Incomes and Nearing Medicare Age. KFF.org. Published March 1, 2019. Accessed September 1, 2020. https:// www.kff.org/health-costs/press-release/ poll-nearly-1-in-4-americans-takingprescription-drugs-say-its-difficultto-afford-medicines-including-largershares-with-low-incomes

2. Winn AN, Keating NL, Dusetzina SB. Factors Associated With Tyrosine Kinase Inhibitor Initiation and Adherence Among Medicare Beneficiaries With Chronic Myeloid Leukemia. J Clin Oncol Off J Am Soc Clin Oncol. 2016;34(36):4323-28. doi:10.1200/JCO.2016.67.4184

3. Curkendall S, Patel V, Gleeson M, et al. Compliance with biologic therapies for rheumatoid arthritis: do patient outof-pocket payments matter? Arthritis Rheum. 2008;59(10):1519-26. doi:10.1002/ art.24114
4. St Charles M, Bollu VK, Hornyak E, et al. Predictors of Treatment Non-Adherence in Patients Treated with Imatinib Mesylate for Chronic Myeloid Leukemia. Blood. 2009;114(22):2209. doi:10.1182/blood. V114.22.2209.2209

5. Bibeau WS, Fu H, Taylor AD, et al. Impact of Out-of-Pocket Pharmacy Costs on Branded Medication Adherence Among Patients with Type 2 Diabetes. J Manag Care Spec Pharm. 2016;22(11):1338-47. doi:10.18553/jmcp.2016.22.11.1338

6. Patient Financial Support. Project HOPE. Published September 14, 2017. Accessed September 1, 2020. doi:10.1377/ hpb20171409.000176

7. Prescription Drugs: Drug Company Programs Help Some People Who Lack Coverage. United States General Accouting Office. Accessed August 29, 2020. https://www.gao.gov/assets/gao01-137.pdf

8. Frerick A. The Cloak of Social Responsibility: Pharmaceutical Corporate Charity. SSRN Electron J. Published November 28, 2016. Accessed September 1, 2020. doi:10.2139/ssrn.2874391

9. Fiscal Totals of the 50 Largest Foundations in the U.S. by Total Giving, 2014. Foundation Center. Published October 2014. Accessed August 26, 2020. http://data.foundationcenter.org/\#/ foundations/all/nationwide/top:giving/ $\underline{\text { list } / 2014}$

10. Global Oncology Trends 2018. IQVIA Institute for Human Data Science. Published 2018. Accessed September 1, 2020. https://www.iqvia. com/-/media/iqvia/pdfs/institutereports/global-oncology-trends-2018. pdf? $=1599593203665$

11. Felder TM, Palmer NR, Lal LS, et al. What is the evidence for pharmaceutical patient assistance programs? A systematic review. J Health Care Poor Underserved. 2011;22(1):24-49. doi:10.1353/hpu.2011.0003
12. Liberati A, Altman DG, Tetzlaff J, et al. The PRISMA statement for reporting systematic reviews and meta-analyses of studies that evaluate healthcare interventions: explanation and elaboration. BMJ. 2009;339(jul21 1):b2700. doi:10.1136/bmj. b2700

13. De Geest S, Zullig LL, Dunbar-Jacob J, et al. ESPACOMP Medication Adherence Reporting Guideline (EMERGE). Ann Intern Med. 2018;169(1):30-35. doi:10.7326/ M18-0543

14. Vrijens B, De Geest S, Hughes DA, et al. A new taxonomy for describing and defining adherence to medications. $\mathrm{Br}$ J Clin Pharmacol. 2012;73(5):691-705. doi:10.1111/j.1365-2125.2012.04167.x

15. Raebel MA, Schmittdiel J, Karter AJ, et al. Standardizing terminology and definitions of medication adherence and persistence in research employing electronic databases. Med Care. 2013;51(8 Suppl 3):S11-21. doi:10.1097/ MLR.0b013e31829b1d2a

16. Peterson AM, Nau DP, Cramer JA, et al. A checklist for medication compliance and persistence studies using retrospective databases. Value Health J Int Soc Pharmacoeconomics Outcomes Res. 2007;10(1):3-12. doi:10.1111/j.1524-4733.2006.00139.x

17. Pluye P, Gagnon M-P, Griffiths F, et al. A scoring system for appraising mixed methods research, and concomitantly appraising qualitative, quantitative and mixed methods primary studies in Mixed Studies Reviews. Int J Nurs Stud. 2009;46(4):529-46. doi:10.1016/j. ijnurstu.2009.01.009

18. Souto RQ, Khanassov V, Hong QN, et al Systematic mixed studies reviews: updating results on the reliability and efficiency of the Mixed Methods Appraisal Tool. Int J Nurs Stud. 2015;52(1):500-01. doi:10.1016/j. ijnurstu.2014.08.010

19. Marrs JC, Saseen JJ. Dyslipidemia control in indigent patients receiving medication assistance compared with insured patients. Pharmacotherapy. 2008;28(5):562-69. doi:10.1592/ phco.28.5.562 
20. Schoen MD, DiDomenico RJ, Connor SE, et al. Impact of the cost of prescription drugs on clinical outcomes in indigent patients with heart disease. Pharmacotherapy. 2001;21(12):1455-63. doi:10.1592/phco.21.20.1455.34473

21. Knott RJ, Petrie DJ, Heeley EL, et al. The effects of reduced copayments on discontinuation and adherence failure to statin medication in Australia. Health Policy Amst Neth. 2015;119(5):620-27. doi:10.1016/j.healthpol.2015.01.003

22. Daugherty JB, Maciejewski ML, Farley JF. The impact of manufacturer coupon use in the statin market. J Manag Care Pharm. 2013;19(9):765-72. doi:10.18553/jmcp.2013.19.9.765

23. Daubresse M, Andersen M, Riggs KR, et al. Effect of Prescription Drug Coupons on Statin Utilization and Expenditures: A Retrospective Cohort Study. Pharmacotherapy. 2017;37(1):12-24. doi:10.1002/phar.1802

24. Wang TY, Kaltenbach LA, Cannon CP, et al. Effect of Medication Co-payment Vouchers on P2Y12 Inhibitor Use and Major Adverse Cardiovascular Events Among Patients With Myocardial Infarction: The ARTEMIS Randomized Clinical Trial. JAMA. 2019;321(1):44-55. doi:10.1001/jama.2018.19791
25. Fanaroff AC, Peterson ED, Kaltenbach LA, et al. Association of a P2Y12 Inhibitor Copayment Reduction Intervention With Persistence and Adherence With Other Secondary Prevention Medications: A Post Hoc Analysis of the ARTEMIS ClusterRandomized Clinical Trial. JAMA Cardiol. 2020;5(1):38-46. doi:10.1001/ jamacardio.2019.4408

26. Seetasith A, Wong W, Tse J, et al. The impact of copay assistance on patient out-of-pocket costs and treatment rates with ALK inhibitors. J Med Econ. 2019;22(5):414-20. doi:10.1080/13696998.2 019.1580200

27. Osterberg L, Blaschke T. Adherence to medication. N Engl J Med. 2005;353(5):487-97. doi:10.1056/ NEJMra050100

28. PQA Measures Overview. Pharmacy Quality Alliance. Published February 27, 2020. Accessed September 2, 2020. https://www.pqaalliance.org/assets/ Measures/2020_PQA_Measures_ Overview.pdf

29. Gellad WF, Thorpe CT, Steiner JF, et al. The myths of medication adherence. Pharmacoepidemiol Drug Saf. 2017;26(12):1437-41. doi:10.1002/pds.4334
30. Grande D. The cost of drug coupons. JAMA. 2012;307(22):2375-6. doi:10.1001/ jama.2012.5603

31. Ross JS, Kesselheim AS. Prescriptiondrug coupons-no such thing as a free lunch. N Engl J Med. 2013;369(13):1188-89. doi:10.1056/NEJMp1301993

32. Erman M, Humer C. U.S. drug prices hit by insurer tactic against copay assistance: analysis. Reuters. https://www.reuters.com/article/ us-usa-healthcare-drugpricing/ us-drug-prices-hit-by-insurer-tacticagainst-copay-assistance-analysisidUSKCN1J2005. Published June 5, 2018. Accessed September 1, 2020.

33. Co-pay Cards FAQs. NeedyMeds. https://www.needymeds.org/copaycards-faqs Published October 21, 2019. Accessed September 2, 2020. 